\title{
Computerized Assessment of the Tumor-stromal Ratio and Proposal of a Novel Nomogram for Predicting Survival in Invasive Breast Cancer
}

\author{
Xu Qian ${ }^{1,2^{*}}$, Feng Xiao $^{3^{*}}$, Yuan-Yuan Chen ${ }^{1,4}$, Jing-Ping Yuan ${ }^{5}$, Xiao-Hong Liu' ${ }^{1,2}$, Lin-Wei Wang1, ${ }^{\bowtie}$, Bin \\ Xiong $1,2 \llbracket$
}

1. Hubei Key Laboratory of Tumor Biological Behaviors, Hubei Cancer Clinical Study Center, Wuhan, China, 430071.

2. Department of Gastrointestinal Surgery, Zhongnan Hospital of Wuhan University, Wuhan, China, 430071.

3. Department of Radiology, Zhongnan Hospital of Wuhan University, Wuhan, China, 430071.

4. Department of Radiation and Medical Oncology, Zhongnan Hospital of Wuhan University, Wuhan, China, 430071.

5. Department of Pathology, Renmin Hospital of Wuhan University, 430060 Wuhan, China.

* These authors contribute equally to manuscript.

$\triangle$ Corresponding authors: Lin-Wei Wang, M.D, Department of Radiation and Medical Oncology, Zhongnan Hospital of Wuhan University, Wuhan, China, 430071. Email: wanglinweiwhu@whu.edu.cn; Bin Xiong, M.D, Department of Gastrointestinal Surgery, Zhongnan Hospital of Wuhan University, Wuhan, China, 430071. Email: binxiong1961@whu.edu.cn.

(1) The author(s). This is an open access article distributed under the terms of the Creative Commons Attribution License (https://creativecommons.org/licenses/by/4.0/). See http://ivyspring.com/terms for full terms and conditions.

Received: 2020.11.11; Accepted: 2021.03.28; Published: 2021.04.19

\begin{abstract}
Background: Various studies have verified the prognostic significance of the tumor-stromal ratio (TSR) in several types of carcinomas using manually assessed H\&E stained histologic sections. This study aimed to establish a computerized method to assess the TSR in invasive breast cancer (BC) using immunohistochemistry (IHC)-stained tissue microarrays (TMAs), and integrate the TSR into a novel nomogram for predicting survival.

Methods: IHC-staining of cytokeratin (CK) was performed in 7 prepared TMAs containing 240 patients with 480 invasive BC specimens. The ratio of tumor areas and stromal areas was determined by the computerized method, and categorized as stroma-low and stroma-high groups using the X-tile software. The prognostic value of the TSR at 5-year disease free survival (5-DFS) in each subgroup was analyzed. Univariate and multivariate analyses were performed and a novel nomogram for predicting survival in invasive breast cancer was established and assessed.

Results: The newly developed computerized method could accurately recognize CK-labeled tumor areas and non-labeled stromal areas, and automatically calculate the TSR. Stroma-low and stroma-high accounted for $38.8 \%(n=93)$ and $61.2 \%(n=147)$ of the cases, according to the cut-off value of $55.5 \%$ for stroma ratio. The Kaplan-Meier analysis showed that patients in the stroma-high group had a worse 5 -DFS compared to patients in the stroma-low group $(P=0.031)$. Multivariable analysis indicated that the T stage, $N$ status, histological grade, ER status, HER-2 gene, and the TSR were potential risk factors of invasive $B C$ patients, which were included into the nomogram $(P<0.10$ for all). The nomogram was well calibrated to predict the probability of 5 -DFS and the C-index was 0.817 , which was higher than any single predictor. A dynamic nomogram was built for convenient use. The area under the curve (AUC) of the nomogram was 0.870 , while that of the TNM staging system was 0.723 . The Kaplan-Meier analysis showed that the nomogram had a better risk stratification for invasive BC patients than the TNM staging system.

Conclusions: Based on IHC staining of CK on TMAs, this study successfully developed a computerized method for TSR assessment and established a novel nomogram for predicting survival in invasive BC patients.
\end{abstract}

Key words: Breast cancer; Tumor stromal ratio; Computerized assessment; Nomogram. 


\section{Introduction}

Breast cancer represents a serious health threat among females worldwide, with an estimated 1.6 million new cases and 520,000 deaths every year according to the GLOBOCAN database [1-2]. Although considerable improvements have been achieved over the past decades due to the progress in screening programmes and comprehensive treatments, the disease prevalence and mortality rates of $\mathrm{BC}$ remained high over the past few decades [3-4]. More prognostic indicators are urgently needed to optimize the risk stratification and contemplate treatment options in invasive BC patients.

Recently, various studies demonstrate that the cancer progression is not only related to biological behavior of tumor cells but also to tumor microenvironment, which includes surrounding blood vessels, the extracellular matrix, other normal cells, and signaling molecules [5-6]. Tumor stroma, as an important component of tumor microenvironment, plays a pivotal role during tumor initiation, progression, and metastasis. Non-malignant cells in the tumor stoma can promote growth and survival of malignant cells through secreting various growth factors, chemokines, and cytokines [7-8]. Therefore, the tumor-stromal ratio, a parameter representing the proportion of tumor-associated stroma, was introduced to the field of cancer research [9-10].

A reliable method for assessment is the basis to explore prognosis of the TSR. In our previous study, CK, an epithelial-specific marker, was applied to specifically label the tumor cells. Compared with H\&E staining, IHC staining of CK resulted in a clear color contrast of brown tumor areas and off-white stromal areas, which made it easier to assess the TSR through visual scoring, and the TSR was proved to be of prognostic value for invasive BC. However, with Cohen's kappa value of 0.77 , the manual method was still criticized due to its intra- and inter-observer variations [11]. To overcome this disadvantage, we established a new computerized method for tumor/stroma recognition and TSR assessment. This method could recognize CK-labeled tumor areas and non-labeled stromal areas, and automatically calculate the TSR.

As a statistical predictive model, a nomogram estimates individualized risk on the basis of clinic-pathologic factors. It assigns relative risk score to each predictor according to its contribution for the prognosis. Owing to its advantage to predict the incidence rate or survival rate through a scoring system rather than calculating a complex formula, the nomogram has emerged as a simpler, yet more advanced method over traditional staging systems [12-13].
Given this, this study developed a computerized method to assess the TSR in invasive BC using CK stained TMAs. Furthermore, a novel nomogram containing the TSR for predicting survival in invasive $\mathrm{BC}$ was established and assessed.

\section{Materials and methods}

\section{Patients and specimens}

The clinical database of $\mathrm{BC}$ of our center has been the data source of several clinical and translational studies [14-15]. From the database, 240 invasive BC specimens were collected and TMAs were constructed according to the same criteria. Major clinic-pathologic characteristics were available, including the tumor stage, location, histological type, lymph node status, ER, PR and HER2 status. TNM staging and histological grading were determined according to the 8th edition of the UICC/AJCC TNM classification [16] and the WHO histological grading [17]. The failure event of the follow-up study was locoregional recurrence or metastasis. 5-year disease-free survival (DFS) was collected from the case file of each patient. Approval of the study protocol was granted by the Institutional Ethics Committee of Zhongnan Hospital of Wuhan University (Scientific Ethical Approval NO.2017057). The study was undertaken according to the ethical standards of the World Medical Association Declaration of Helsinki.

\section{Tissue microarrays construction}

TMAs were constructed using standard procedures in collaboration with Shanghai Outdo Biotech Co. Ltd. (Shanghai, China), as previously described [18]. For all specimens, diagnostic $4 \mu \mathrm{m}$ H\&E-stained sections were obtained and inspected, and the most invasive tumor areas containing both tumor cells and tumor stroma were identified. Corresponding areas were marked on the original FFPE block for cutting. Two cores were taken from marked areas of each paraffin block using punch cores. The cores were then deposited into recipient paraffin blocks with 70 cylinders. Seven TMAs blocks containing 480 cores were constructed and cut into $4 \mu \mathrm{m}$ sections, with one slide every 50 retained for H\&E staining and quality control.

\section{IHC staining of CK}

IHC staining of CK was performed in our previous study [11]. Firstly, TMA slides were deparaffinized and rehydrated with successive washes in dimethylbenzene (15min) and alcohol ((100, 90 , and $70 \%, 5 \mathrm{~min}$ each). Epitope retrieval was performed using $0.01 \mathrm{~mol} / \mathrm{L}$ citrate buffer ( $\mathrm{pH}$ 6.0) heated by a microwave oven $\left(95^{\circ} \mathrm{C}, 15 \mathrm{~min}\right)$. The 
endogenous peroxidase activity was blocked using $3 \% \mathrm{H} 2 \mathrm{O} 2(10 \mathrm{~min})$ at room temperature. The blocking antibody ( $2 \%$ BSA) was applied to decrease background intensity. All slides were incubated with the anti-pan CK antibody (mouse anti-human, ZSGB-BIO, China, clone AE1/AE3; 1:100 dilution) overnight at $4^{\circ} \mathrm{C}$, and then with corresponding secondary antibody (1:250 dilution) for $30 \mathrm{~min}$ at 37 ${ }^{\circ} \mathrm{C}$. Diaminobenzidine was added as the chromogen and counterstaining was performed with hematoxylin for $2 \mathrm{~min}$. Following dehydration, the slides were sealed with resin mount.

\section{Image acquisition and assessment of TSR}

TMAs were scanned with the Aperio VERSA automated slide scanner (Leica Biosystems Imaging, Buffalo Grove, IL). Digital images of cores in TMAs were obtained using the Aperio Image Scope Software (Leica Biosystems Imaging, Buffalo Grove, IL). Using a computerized TSR assessment approach, digital images of cores in TMAs were assessed to calculate the proportion of stroma areas. The computerized approach comprises the following steps, (1)Transforming the color image into grayscale image; (2) Calculating the image gradient using edge and sobel operators to detect the contours of objects; (3) Obtaining the tumor objects in the image using morphological operation (dilate-> fill-> erode) with small objects eliminated; (4) Staining the tumor objects and non-tumor areas in the image with different colors (colored image); 5)Increasing the image contrast differences using histogram equalization for subsequent segmentation; 6Performing image segmentation using otsu algorithm; (7)Obtaining the whole core object in the image (mask map); (8) Merging the colored image and mask map to get the final image and calculating the TSR, TSR=area of stroma objects (in cyan)/area of the whole core object (tumor objects (in magenta) + stromal objects (in cyan)). The field of highest stromal percentage from two cores of each specimen were considered crucial.

\section{Statistical analysis}

The cut-off point of TSR was determined using the X-tile software based on the best $P$ value principle. Distribution of the clinic-pathologic factors between stroma-low and stroma-high groups was evaluated using Pearson $\chi^{2}$ test or Fisher's exact test. The Kaplan-Meier method was performed to analyze the 5-DFS. The log-rank test was applied for comparison between the curves. Unadjusted HRs (hazard ratios) and $95 \%$ CIs of TSR for 5-DFS in each subgroup were calculated using by Cox proportional hazard analysis. The Cox regression model was used to perform univariate and multivariate analyses for 5-DFS. In the univariate analysis, potential risk factors were selected. In the multivariate analysis, three selection procedures (enter, forward, backward) were performed to select variables into the best-fit model. A statistical significance level of 0.10 was used, which can reduce the impact caused by the small sample size. The above statistical analyses were performed using IBM SPSS statistics (version 23.0 for Windows).

Based on the results of multivariate regression and clinical experience, a baseline nomogram was constructed to generate probability of 5-DFS. The performance of the nomogram included its discrimination and calibration. Discrimination was defined as a model's ability to correctly distinguish non-events and events, which was quantified using a concordance index (C-index). Calibration measures how closely the predicted survival rate agree with the actual survival rate, which was evaluated by the calibration plot. In addition, the receiver operating characteristic (ROC) curve was used to compare the discrimination ability of the nomogram with the TNM staging system. The Kaplan-Meier curve and the Log-rank test was performed to estimate the probability of 5-DFS between risk subgroups. The above statistical analyses were performed using $\mathrm{R}$ 3.6.3 software (https://cran.r-project.org/). The dynamic nomogram was built through package "DynNom".

\section{Results}

\section{IHC staining images and computerized assessment results}

Typical IHC staining images and corresponding computerized assessment results are shown in Fig. 2. IHC staining of CK makes a strong color contrast of brown tumor areas and off-white stromal areas (A1 and B1). After computerized recognition, tumor areas were marked in magenta, stromal areas in cyan, and none-cell areas in black (A2 and B2). Panels A1, A2 are examples of high stroma, with an estimated TSR of 78.1\%. Panels B1, B2 are examples of low stroma, with an estimated TSR of $37.9 \%$.

\section{Evaluation of tumor-stromal ratio}

As the TSR quantified by the computerized method was a continuous variable, the X-tile software based on the best $P$ value principle was adopted to identify the optimal cut-off point of TSR. A cut-off point of $55.5 \%$ was used to categorize patients into stroma-low (TSR $\leq 55.5 \%$ ) and stroma-high (TSR > $55.5 \%$ ) groups. Among 240 specimens, $38.8 \%$ were determined as stroma-low and $61.2 \%$ as stroma-high. 


\section{Correlation between TSR and major clinic- characteristics}

There were 240 invasive $\mathrm{BC}$ patients included in the study. The median age was 48 years (range, 29-78 years) at the date of surgery. Table 1 listed the major clinic-pathological characteristics stratified for stroma-low and stroma-high groups. The TSR was significantly associated with menopausal status $(P=$ $0.031)$, but not with age $(P=0.244)$, histological type $(P=0.514), \mathrm{T}$ stage $(P=0.629), \mathrm{N}$ status $(P=0.205)$, histological grade $(P=0.622)$, ER status $(P=0.277), \mathrm{PR}$ status $(P=0.499)$, and HER2 gene status $(P=0.090)$ (Table 1).

Table 1. The relationship between TSR and major clinico-pathological characteristics.

\begin{tabular}{|c|c|c|c|c|}
\hline Characteristics & $\begin{array}{l}\text { Total, } \mathrm{n} \\
(\%)\end{array}$ & $\begin{array}{l}\text { Stroma-high, } \mathrm{n} \\
(\%)\end{array}$ & $\begin{array}{l}\text { Stroma-low, n } \\
(\%)\end{array}$ & $\begin{array}{l}P \\
\text { value }\end{array}$ \\
\hline Age (years) & & & & 0.244 \\
\hline$\leq 50$ & $149(62.1)$ & 87 (59.2) & $62(66.7)$ & \\
\hline$>50$ & $91(37.9)$ & $60(40.8)$ & $31(33.3)$ & \\
\hline Menopausal status & & & & 0.031 \\
\hline Premenopausal & $134(55.8)$ & $74(50.3)$ & $60(64.5)$ & \\
\hline Postmenopausal & $106(44.2)$ & $73(49.7)$ & $33(35.5)$ & \\
\hline \multicolumn{5}{|l|}{ Histological type } \\
\hline $\begin{array}{l}\text { Invasive ductal } \\
\text { carcinoma }\end{array}$ & $191(79.6)$ & $115(78.2)$ & $76(81.7)$ & 0.514 \\
\hline Others & $49(20.4)$ & $32(21.8)$ & 17(18.3) & \\
\hline$T$ stage $(\mathrm{cm})$ & & & & 0.629 \\
\hline $\mathrm{T} 1(\mathrm{~T} \leq 2)$ & $35(15.0)$ & $19(12.9)$ & $16(17.2)$ & \\
\hline $\mathrm{T} 2(2<\mathrm{T} \leq 5)$ & $162(67.5)$ & $102(69.4)$ & $60(64.5)$ & \\
\hline $\mathrm{T} 3(\mathrm{~T}>5)$ & $43(17.5)$ & $26(17.7)$ & $17(18.3)$ & \\
\hline $\mathrm{N}$ status & & & & 0.205 \\
\hline $\mathrm{N}$ negative & $109(45.4)$ & $62(42.2)$ & $47(50.5)$ & \\
\hline $\mathrm{N}$ positive & $131(54.6)$ & $85(57.8)$ & $46(49.5)$ & \\
\hline Histological grade & & & & 0.622 \\
\hline I & $40(16.7)$ & $25(17.0)$ & $15(16.1)$ & \\
\hline II & $141(58.8)$ & $83(56.5)$ & $58(62.4)$ & \\
\hline III & $59(24.6)$ & $39(26.5)$ & $20(21.5)$ & \\
\hline ER status ${ }^{a}$ & & & & 0.277 \\
\hline Positive & $106(44.2)$ & $69(46.9)$ & $37(39.8)$ & \\
\hline Negative & $134(55.8)$ & $78(53.1)$ & $56(60.2)$ & \\
\hline PR status ${ }^{a}$ & & & & 0.499 \\
\hline Positive & 107 (44.6) & $63(42.9)$ & $44(47.3)$ & \\
\hline Negative & $133(55.4)$ & $84(57.1)$ & $49(52.7)$ & \\
\hline \multicolumn{5}{|l|}{ HER2 gene ${ }^{b}$} \\
\hline Amplification & $51(21.3)$ & $26(17.7)$ & $25(26.9)$ & 0.090 \\
\hline Non-amplification & 189 (78.7) & $121(82.3)$ & 68 (73.1) & \\
\hline $\begin{array}{l}{ }^{a} E R, P R \text { was determine } \\
\text { [19]; bHER2 gene was } \\
\text { according to guideline } \\
\text { tumor-stromal ratio; E } \\
\text { receptor-2. }\end{array}$ & y immunoh & $\begin{array}{l}\text { toptor; HER2: hun } \\
\text { t cancer; T: tumo }\end{array}$ & $\begin{array}{l}\text { ing according to g } \\
\text { ybridization (FISI } \\
\text { J: node; TSR: } \\
\text { epidermal grow }\end{array}$ & $\begin{array}{l}\text { uideline } \\
\text { I) factor }\end{array}$ \\
\hline
\end{tabular}

\section{Prognosis of BC patients according to TSR}

For the entire patient cohort $(n=240)$, the 5-year disease free survival rate was $62.0 \%$. As expected, those conventional factors were associated with invasive BC patients' 5-DFS $(P<0.05$ for all $)$ (Supplementary Table 1), including T stage, N status, histological grade, histological type, ER, PR, HER-2 status, and menopausal status. The survival curve of patients with high or low TSR are shown in Fig. 3. A worse DFS was found for patients with high stroma as compared to patients with low stroma $(\chi 2=4.659, P=$ 0.031 ), with 5-year disease free survival rate of, respectively, 56.5 vs. $71.0 \%$. The result indicated that the TSR might be a prognostic predictor for invasive $\mathrm{BC}$, and patients with stroma-rich tumors showed a trend toward a worse outcome.

\section{Subgroup analysis of the TSR associated with 5-DFS}

Prognostic significance of the TSR for 5-DFS was analyzed in each subgroup (Fig. 4). For the whole cohort, a better 5-DFS was found for patients with low stroma as compared to patients with high stroma (HR $0.61 ; 95 \%$ CI $0.38-0.99 ; P=0.034)$. Subgroup analysis demonstrated that the TSR was significantly associated with 5-DFS in invasive ductal carcinoma (HR 0.61; 95\% CI 0.38-0.99; $P=0.045$ ), $\mathrm{N}$ positive (HR $0.57 ; 95 \%$ CI $0.34-0.97 ; P=0.037$ ), ER negative (HR $0.43 ; 95 \%$ CI $0.25-0.74 ; P=0.002)$ and HER2 gene non-amplification groups (HR 0.51; 95\% CI 0.28-0.90; $P=0.021)$. In addition, a non-significant correlation between low stroma and better 5-DFS was observed $(P>0.05)$ in groups of age, menopausal status, histological grade, and PR status. Notably, HR of the T1 stage and histological grade I groups had a very broad the confidence interval, probably caused by the relatively small sample size or wide sample variability.

Table 2. Multivariable analysis for 5-DFS and the C-index for single predictors.

\begin{tabular}{|c|c|c|c|c|}
\hline \multirow[t]{2}{*}{ Parameters } & \multicolumn{3}{|c|}{ Multivariable analysis } & \multirow[t]{2}{*}{ C-index $(95 \% \mathrm{CI})$} \\
\hline & HR & $95 \% \mathrm{CI}$ & P valuea & \\
\hline T stage $(\mathrm{cm})$ & & & & $0.625(0.576-0.675)$ \\
\hline $\mathrm{T} 1(\mathrm{~T} \leq 2)$ & 1.000 & & & \\
\hline $\mathrm{T} 2(2<\mathrm{T} \leq 5)$ & 2.576 & $0.925-7.171$ & 0.070 & \\
\hline $\mathrm{T} 3(\mathrm{~T}>5)$ & 3.506 & $1.199-10.251$ & 0.022 & \\
\hline $\mathrm{N}$ status & & & & $0.678(0.635-0.721)$ \\
\hline Negative & 1.000 & & & \\
\hline Positive & 3.854 & $2.201-6.747$ & $<0.001$ & \\
\hline Histological grade & & & & $0.684(0.639-0.729)$ \\
\hline I & 1.000 & & & \\
\hline II & 1.479 & $0.513-4.262$ & 0.469 & \\
\hline III & 4.250 & $1.413-12.782$ & 0.010 & \\
\hline ER status & & & & $0.611(0.563-0.659)$ \\
\hline Negative & 1.000 & & & \\
\hline Positive & 0.568 & $0.325-0.995$ & 0.048 & \\
\hline \multicolumn{5}{|l|}{ PR status } \\
\hline Negative & 1.000 & & & \\
\hline Positive & 1.076 & $0.640-1.807$ & 0.783 & \\
\hline HER2 gene & & & & $0.588(0.541-0.635)$ \\
\hline Non-amplification & 1.000 & & & \\
\hline Amplification & 2.045 & $1.258-3.325$ & 0.004 & \\
\hline TSR & & & & $0.555(0.505-0.605)$ \\
\hline Stroma-high & 1.000 & & & \\
\hline Stroma-low & 0.643 & $0.399-1.035$ & 0.069 & \\
\hline
\end{tabular}

T: tumor; N: node; TSR: tumor-stromal ratio; ER: estrogen receptor; PR:

progesterone receptor; HER2: human epidermal growth factor receptor-2. 


\section{Univariable and multivariable analysis of the TSR and other parameters}

The relationship between all cliniccharacteristics and 5-DFS was investigated in univariate analyses. Factors that were included into the multivariate model predicting 5-DFS were $\mathrm{T}$ stage $(P<0.001)$, N status $(P<0.001)$, histological grade $(P<$ $0.001)$, ER status $(P<0.001)$, PR status $(P=0.006)$, HER2 gene $(P<0.001)$, and the TSR $(P=0.034)$. Factors that remained in the multivariate model for the construction of nomogram were $\mathrm{T}$ stage, $\mathrm{N}$ status, histological grade, ER status, HER2 gene, and the TSR $(P<0.10$ for all) (Table 2$)$.

\section{Construction and validation of the prognostic nomogram}

Based on the results of multivariate regression and clinical experience, a nomogram was constructed to predict the probability of disease-free survival at the follow-up of 5 years for invasive BC patients (Fig. 5 ). According to the contribution of each predictor for the prognosis (scaled by the proportional regression coefficient of each predictor), relative risk score was assigned to each predictor [12]. When using the nomogram, an upward vertical line was drawn from the covariate to the points bar to calculate points. The detailed points of each variable were as follows, $\mathrm{T}$ stage (T1: 0.0, T2: 65.1, T3: 87.0), $\mathrm{N}$ status (negative: 0.0. positive: 93.4), histological grade (I: 0.0, II: 27.3, III: 100.0), ER status (positive: 0.0, negative: 36.34), HER-2 gene (negative: 0.0, positive: 49.6), and the TSR (stroma-low: 0.0, stroma-high: 30.8). Based on the sum of the covariate points, a downward vertical line was drawn to confirm the probability of 5-DFS. Furthermore, the dynamic version of this nomogram (Fig. S1) was established, which could assist users in obtaining probability of disease-free survival at any time within 60 months. The C-index of the nomogram was 0.817 (95\% CI: 0.775-0.858), which indicated a good discriminative ability. The calibration plot for predicting 5-year DFS probability also showed favorable consistency between nomogram predictions and observed outcomes (Fig. 6A). In addition, we compared the predictive ability of the nomogram with included independent prognostic factors. The C-indices of T stage, $\mathrm{N}$ status, histological grade, ER status, HER2 gene and TSR were significantly lower than the C-index of the nomogram $(0.817,95 \% \mathrm{CI}$ : 0.775-0.858) (Table 2).

\section{Comparison of in the predictive value of the nomogram and TNM staging system}

The nomogram had a better ability to predict recurrence as compared to TNM staging system $(P<$ 0.001), with an AUC of, respectively, 0.870 (95\% CI:
0.823-0.917) vs. 0.723 (95\% CI: 0.657-0.790) (Fig. 6B). Meanwhile, the sum of the covariate points was calculated for each patient. Through the X-tile software, optimum cut-off points of the total points for three groups were identified (222.2, 316.8). Patients with total points $\leq 222.2,222.2<$ total points $\leq$ 316.8 , and total points $>316.8$ were categorized as I $(\mathrm{n}=144)$, II $(\mathrm{n}=64)$, and III $(\mathrm{n}=32)$ groups, respectively. Kaplan-Meier curves showed that the nomogram had a better ability to distinguish BC patients into three groups with different prognoses $\left(\chi^{2}=128.361, P<\right.$ 0.001 for I/II and II/III) than the TNM staging system $(\chi 2=59.657, P=0.015$ for I/II, $P<0.001$ for II/III) (Fig. $7)$.

\section{Discussion}

The tumor stroma consists of multiple components, which play a vital role in the tumor progression. TSR, as a new parameter which represents the amount of tumor-associated stroma, has been proved to be significant in prognosis evaluation of different cancer types [21-23]. Our data indicated that the TSR might be a prognostic predictor for invasive $\mathrm{BC}$, and patients with stroma-rich tumors showed a trend toward a worse outcome.

Accurate assessment of the TSR is the key to appreciating its prognostic value. Currently, the TSR is largely assessed in the hematoxylin-eosin (HE) staining section with two methods, visual eyeballing [21-22] and point counting [23-24]. Visual eyeballing is a manual method with two-steps to determine the TSR. Firstly, at low magnification, the most invasive tumor areas were selected. Then, at high magnification, image fields containing both stroma and tumor are assessed and TSR is scored per tenfold percentage. The other is a semi-automated point counting method. A sample of 300 random points are superimposed on the selected area. Under each of the 300 points, the histopathology is categorized as 'tumor,' 'stroma,' or 'unclassified (necrosis, blood vessels, inflammation, blank areas).' The TSR is expressed as the proportion of 'stroma' under the 300 points, compared with all points per section.

These two methods have been applied in various studies, which assessed TSR using HE staining slides and could be easily performed in routine pathology diagnostics [25-26]. However, sometimes the boundary of tumor nests cannot be accurately identified due to low contrast between the tumor and stroma in HE staining, which makes it difficult for observers to perform the TSR assessment. In our previous study, IHC staining of CK was utilized to make a clear color contrast of brown tumor areas and off-white stromal areas, which makes it easier to assess TSR through visual scoring. However, with 
Cohen's kappa value of 0.77 , the manual method was still criticized due to its intra- and inter-observer variations [11]. To improve this disadvantage, we developed a computerized tumor/stroma recognition method. The computerized method with optimal reproducibility could enable the objective and standardized TSR quantification. The procedures were briefly demonstrated in Fig. 1C. Two major steps were performed to recognize the tumor object and the whole core object, respectively. In the final image, tumor areas were marked in magenta, stromal areas in cyan, and none-cell areas in black. TSR was automatically calculated as the area of stroma divided by the area of the whole core (tumor and stroma). Compared with similar studies of computer aided-recognition methods [27-29], several technical improvements have been made in our study. Firstly, as mentioned above, the key for computerized TSR assessment is to identify the boundary of tumor nests more accurately. To maximally differentiate any obscure boundaries, edge and sobel operators were utilized to make the calculated gradient value more accurate, so as to get clear contours of tumor objects. Secondly, the cell nuclei of stroma cells are stained in blue by hematoxylin, which may be mistakenly identified as tumor objects. To prevent this, in the morphological operation (dilate-> fill-> erode), a suitable threshold was set to eliminate small objects like the cell nuclei. Comparison of recognition results by different thresholds are shown in Fig. 8. Thirdly, combing with a high throughput approach, massive digital images of cores in TMAs could be assessed to calculate the proportion of stromal areas. It eliminated the onerous time and workflow required by visual scoring performed by experienced pathologists. In addition, this computerized tumor/stroma recognition method can also be applied to TSR assessment on microscopic fields under 10x objective or 20x objective, which is shown in Fig. S2. In routine pathology diagnostics, evaluation of the TSR started with microscopical orientation at low magnification. Subsequently, a high magnification was used in the selected areas. Through computerized assessment, the TSR can be accurately determined, rather than scored per tenfold increments.

So far, various studies have reported the prognostic value of TSR in different types of $B C$, for example, in triple-negative BC [30-31], lymph node-negative $\mathrm{BC}$ [32], primary operable invasive ductal BC [33], estrogen receptor-positive BC [24], and inflammatory BC [26]. Most of them have shown an association between high stroma content and a poor prognosis. This study aimed at exploring the prognostic value of the TSR in invasive BC using CK-staining TMAs and computerized assessment. Consistent with previous studies from central Chinese [34-35], there was a high proportion of patients with lymph node-positivity and hormone receptornegativity, which indicated more patients with aggressive $\mathrm{BC}$ and less patients qualified for endocrine therapy. As a result, prognosis of the subjects is poorer than that of common patients with invasive breast cancer. Among 240 specimens, 38.8\% were determined as stroma-low and $61.2 \%$ as stroma-high through computerized assessment, and a worse DFS was found for patients with high stroma as compared to patients with low stroma $(\chi 2=4.659, P=$ 0.031 ), with 5-year disease free survival rate of, respectively, 56.5 vs. $71.0 \%$. Subgroup analysis demonstrated that the TSR was significantly associated with 5-DFS in invasive ductal carcinoma, $\mathrm{N}$ positive, ER negative, and HER-2 gene non-amplification groups.

The gold standard for prognostication in oncology remains the TNM staging system. It creates a system with a finite number of stages. For an individual patient, a higher TNM stage corresponds to a worse prognosis, but a concrete incidence rate or survival rate cannot be immediately determined. In addition, patients with the same anatomical spread yet variable outcomes (recurrence or survival) are categorized into the same stage, and have the same prognosis, which results in heterogeneity [36-37]. Correspondingly, the nomogram, as a statistical predictive model, has emerged as a simpler, yet more advanced method [38-39]. It assigns relative risk score to each predictor according to its contribution for the prognosis, and predicts the incidence rate or survival rate through a scoring system. Through the univariate and multivariate Cox regression analysis, variables including $\mathrm{T}$ stage, $\mathrm{N}$ status, histological grade, ER status, HER2 gene and TSR were selected into the best-fit model. The clinical availability of these six variables were examined, and then a nomogram was constructed to predict the probability of disease-free survival at the follow-up of 5 years for invasive BC patients. The nomogram showed favorable predictive performance, which included the discrimination ability quantified by the C-index $(0.817,95 \%$ CI: $0.775-0.858$ ) and the calibration ability evaluated by the calibration plot. In addition, the results of C-index and AUC demonstrated that the predictive performance of the nomogram was superior to included independent prognostic factors and the TNM staging system. 

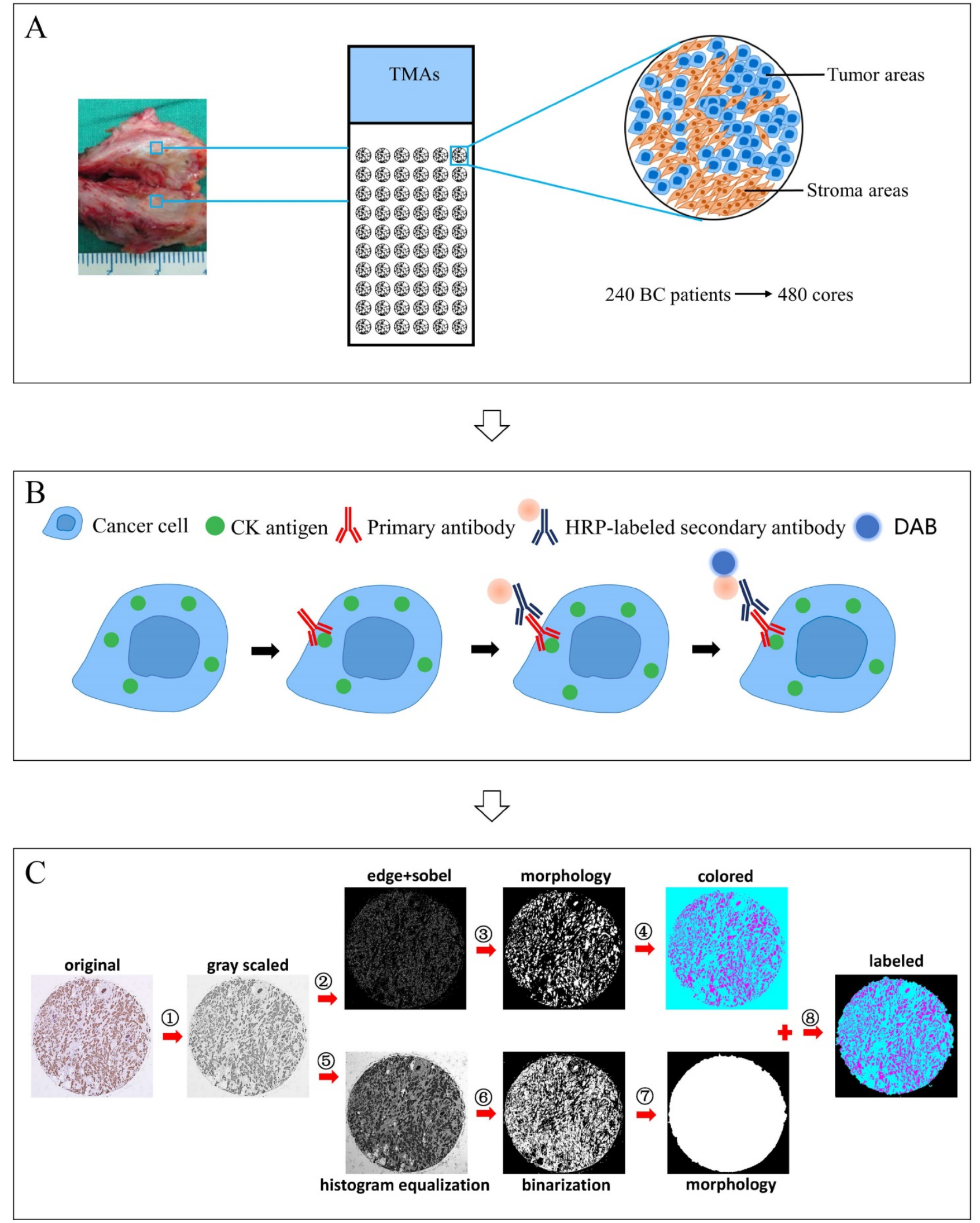

Figure 1. Major technical procedures of this study. Panel A, TMAs with 480 cores were constructed using 240 cases of breast cancer specimens. Panel B, IHC staining of CK was performed. Panel C, computerized TSR assessment was performed, involving the following steps, (1) Transforming the color image into grayscale image; (2) Calculating the image gradient using edge and sobel operators to detect the contours of objects; (3)Obtaining the tumor objects in the image using morphological operation (dilate-> fill-> erode) with small objects eliminated; (4) Staining the tumor objects and non-tumor areas in the image with different colors (colored image); 5 Increasing the image contrast differences using histogram equalization for subsequent segmentation; (6)Performing image segmentation using otsu algorithm; (7)Obtaining the whole core object in the image (mask map); (8) Merging the colored image and mask map to get the final image and calculating the TSR, TSR=area of stroma objects (in cyan)/area of the whole core object (tumor objects (in magenta) + stromal objects (in cyan)). 

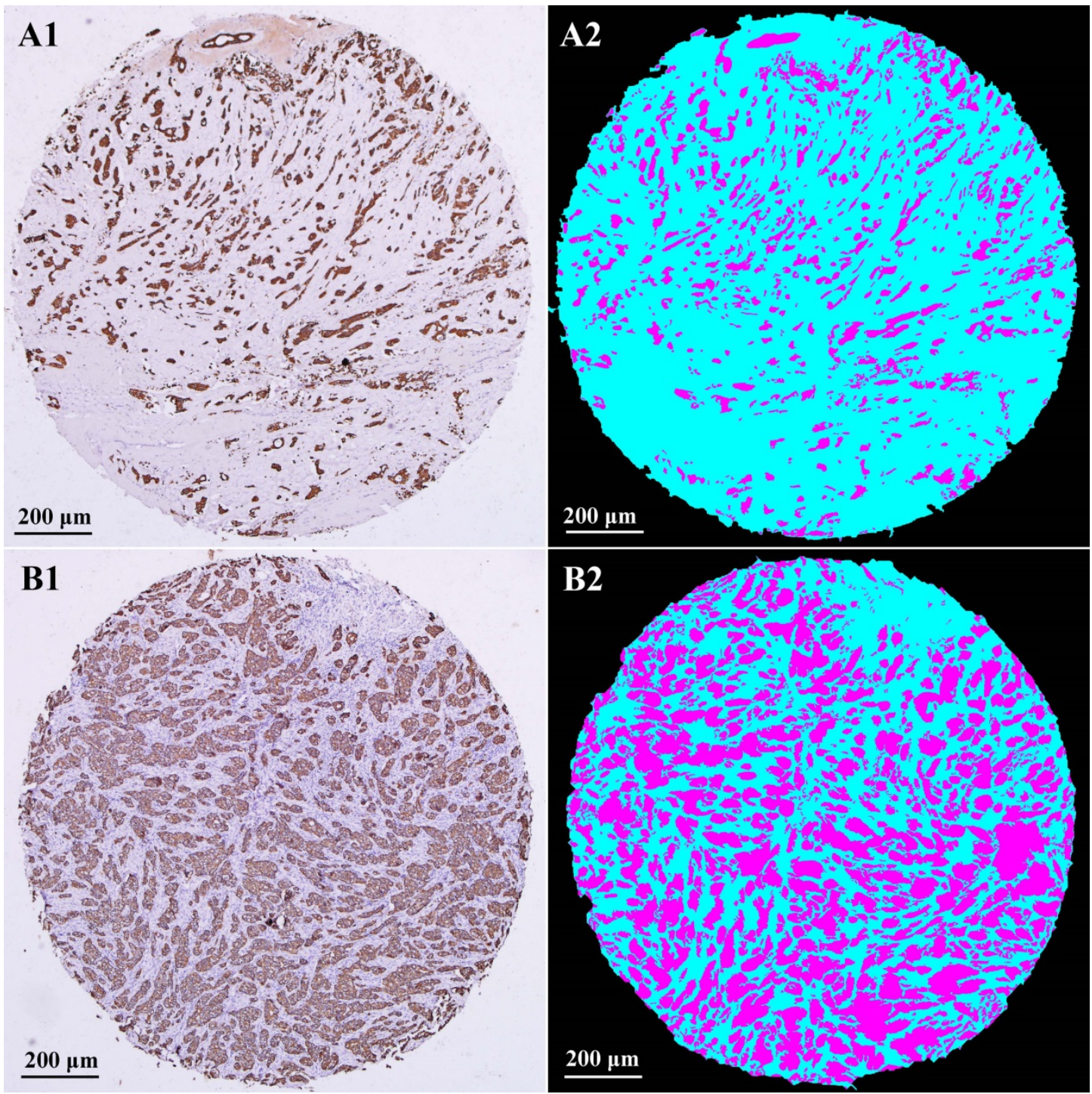

Figure 2. IHC staining images and computerized assessment results in TMAs. IHC staining of CK could specifically label tumor areas with clear contrast (A1 and B1). After computerized recognition, tumor areas were marked in magenta, stromal areas in cyan, and none-cell areas in black (A2 and B2). Examples of high stroma (A1 and A2); Examples of low stroma (B1 and B2). IHC: immunohistochemistry; CK: cytokeratin; TSR: tumor-stromal ratio.

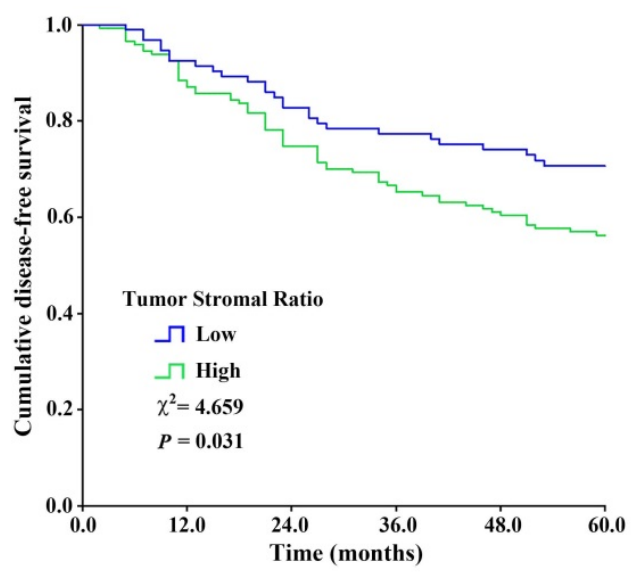

Figure 3. The Kaplan-Meier survival curve showing disease-free survival after stratification by TSR. High stroma was associated with worse 5-year disease free survival $\left(X^{2}=4.659, P=0.031\right)$ compared with low stroma.
The ability to categorize patients into different risk groups accurately is equally important because this is the premise of formulating treatment strategies. As a result, the sum of the covariate points was calculated for each patient, and a risk stratification based on the nomogram was established. Compared with the TNM staging system $\left(X^{2}=59.657, P=0.015\right.$ for I/II, $P<0.001$ for II/III), the nomogram had a better ability to distinguish BC patients into three groups with different prognoses $\left(X^{2}=128.361, P<0.001\right.$ for I/II and II/III). Given these statistical results, we believed that this nomogram had adequate power of discrimination, calibration, and satisfactory risk stratification.

However, there are still several limitations in our study. Firstly, it is a retrospective research with a relatively small sample capacity, which may compromise the predictive performance of the 
nomogram. It will be valuable to conduct a prospective study with a larger sample. Secondly, although TMAs were constructed using standard procedures that only the most invasive tumor areas containing both tumor cells and tumor stroma were identified, not every core of TMAs can completely represent the optimal site for TSR assessment. More tumor cores taken from each specimen may reduce the selection bias. Thirdly, available external data for external validation of the nomogram is lacked. A supplementary of external data can help evaluate the external applicability of the nomogram.

\section{Conclusions}

In general, our study established a computerized method to automatically assess the TSR in invasive $\mathrm{BC}$ using $\mathrm{CK}$ stained TMAs, and demonstrates that invasive BC patients of low TSR have poor prognoses. Furthermore, a nomogram containing TSR for predicting survival in invasive $\mathrm{BC}$ was established and assessed, which provided a comprehensive individualized risk prediction strategy, and might assist clinicians to make optimal care decisions for BC patients.

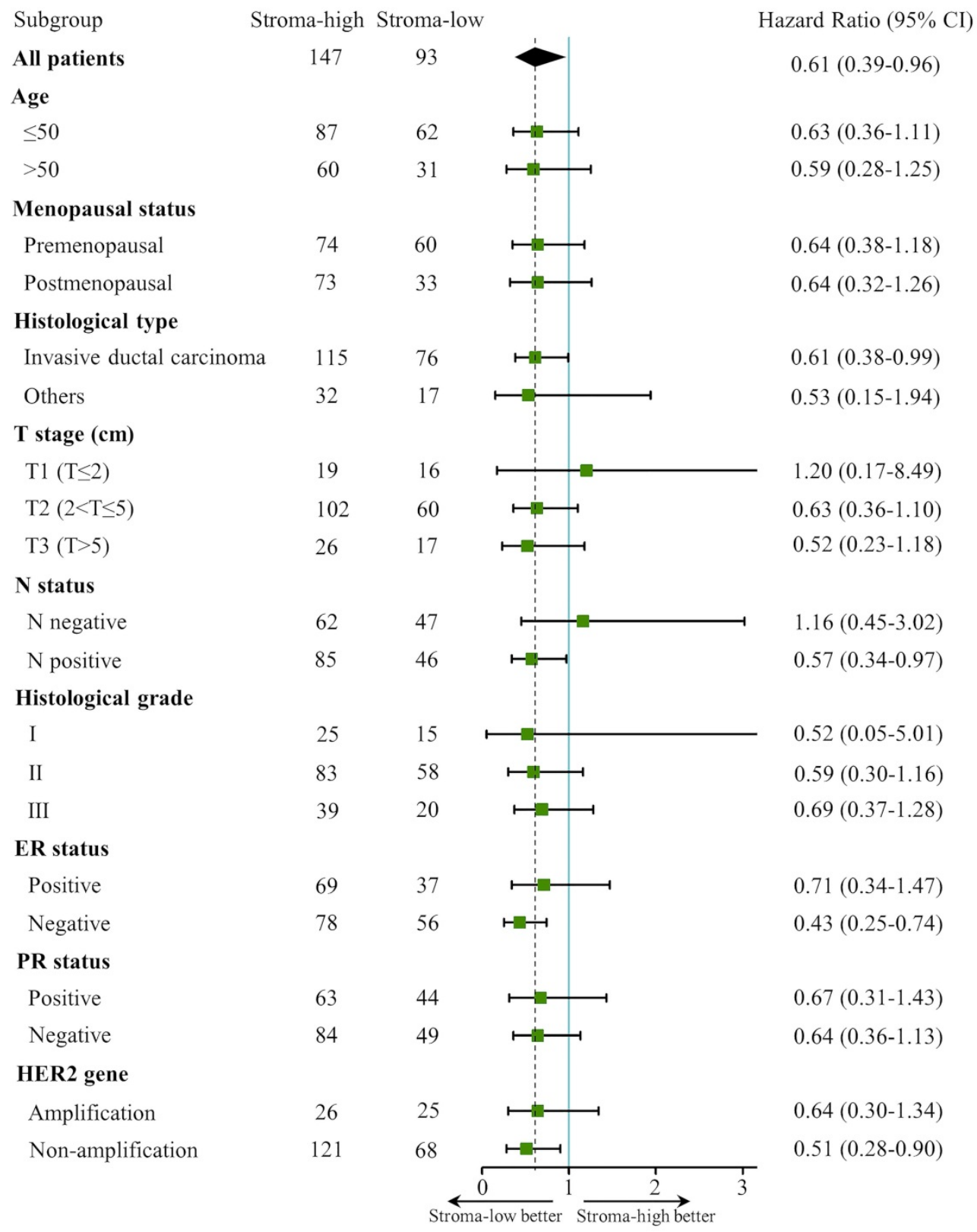

Figure 4. The forest plot of TSR associated with 5-DFS in subgroups. The dashed line represents the hazard ratio 0.61 in all patients. Cl: confidence interval. 
Points

T stage

$\mathrm{N}$ status

Histological grade

ER status

HER-2 gene

TSR

Total Points

5-year DFS Probability

$\begin{array}{lllllllllll}0 & 10 & 20 & 30 & 40 & 50 & 60 & 70 & 80 & 90 & 100\end{array}$

$\mathrm{T} 2$

$\mathrm{T} 12^{\mathrm{T} 3}$ Positive

Negative

I Negative III

Positive

Negative Positive

Stroma-low

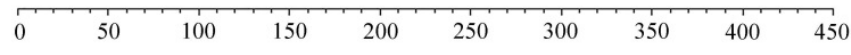

$\begin{array}{lllllllllllllllll}0.95 & 0.9 & 0.85 & 0.80 .750 .7 & 0.6 & 0.5 & 0.4 & 0.3 & 0.2 & 0.10 .05\end{array}$

Figure 5. Nomogram predicting 5-year DFS probability of invasive BC patients.

A

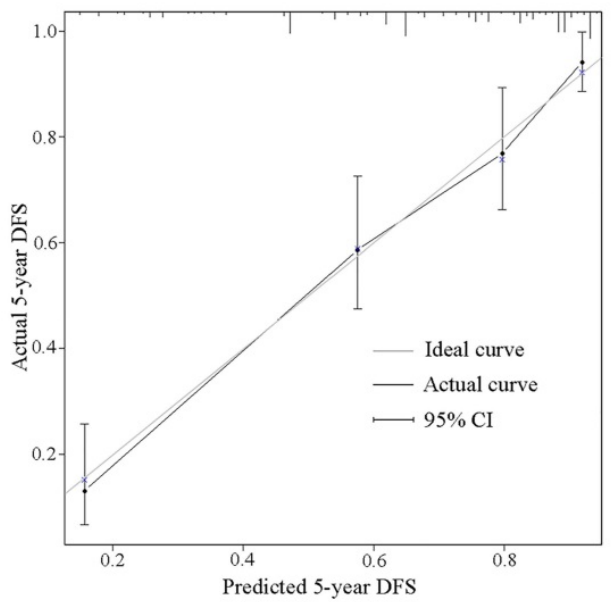

B

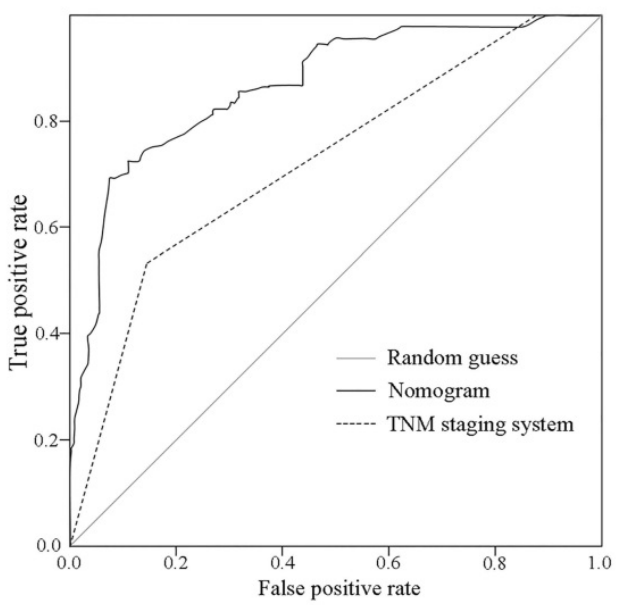

Figure 6. Calibration plot and ROC curve of the nomogram. A. On the calibration plot, the grey line represents an ideal evaluation, whereas the black line represents the performance of the nomogram, which showed favorable agreement between the predicted rate and actual rate. B. On the ROC curve, AUC of the nomogram $(0.870(95 \% \mathrm{Cl}$ : $0.823-0.917))$ is greater than the TNM staging system $(0.723(95 \% \mathrm{Cl}: 0.657-0.790))$.

A

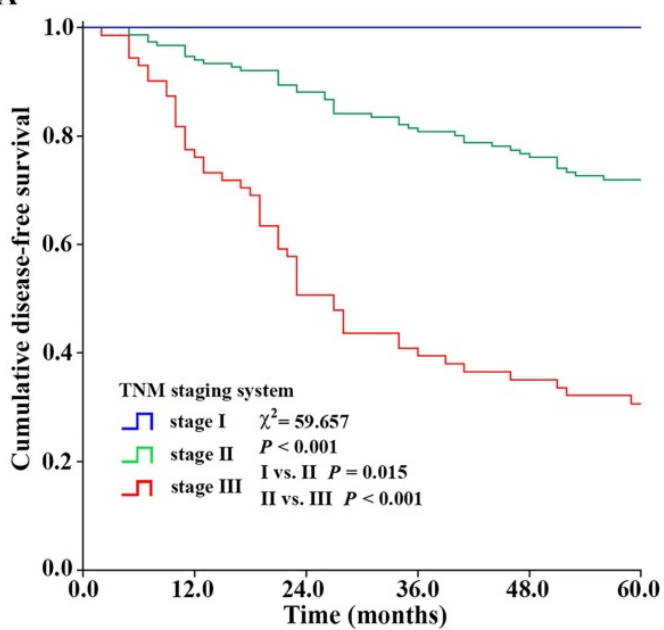

B

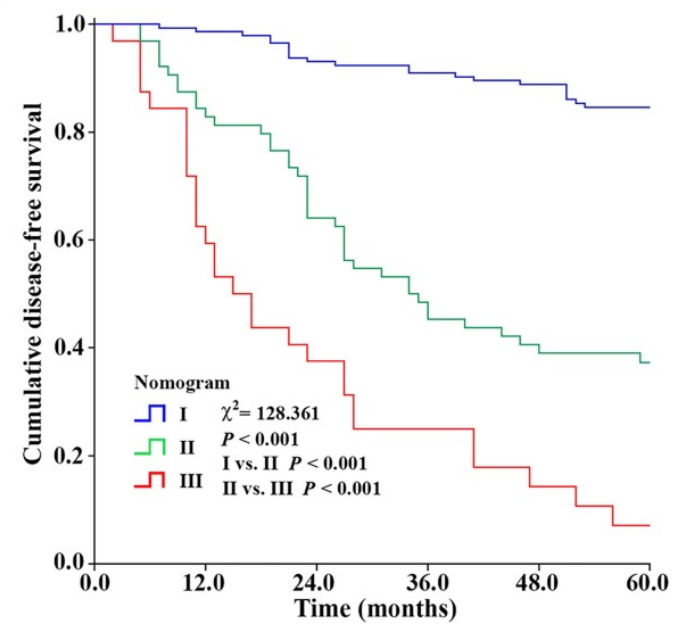

Figure 7. Kaplan-Meier survival curves showing the stratification of the nomogram and TNM staging system. The nomogram had a better ability to distinguish BC patients into three groups with different prognoses $\left(X^{2}=128.361, P<0.001\right.$ for $I / I I$ and II/III) than the TNM staging system $\left(X^{2}=59.657, P=0.015\right.$ for $\mathrm{I} / \mathrm{II}, P<0.001$ for II/III). 

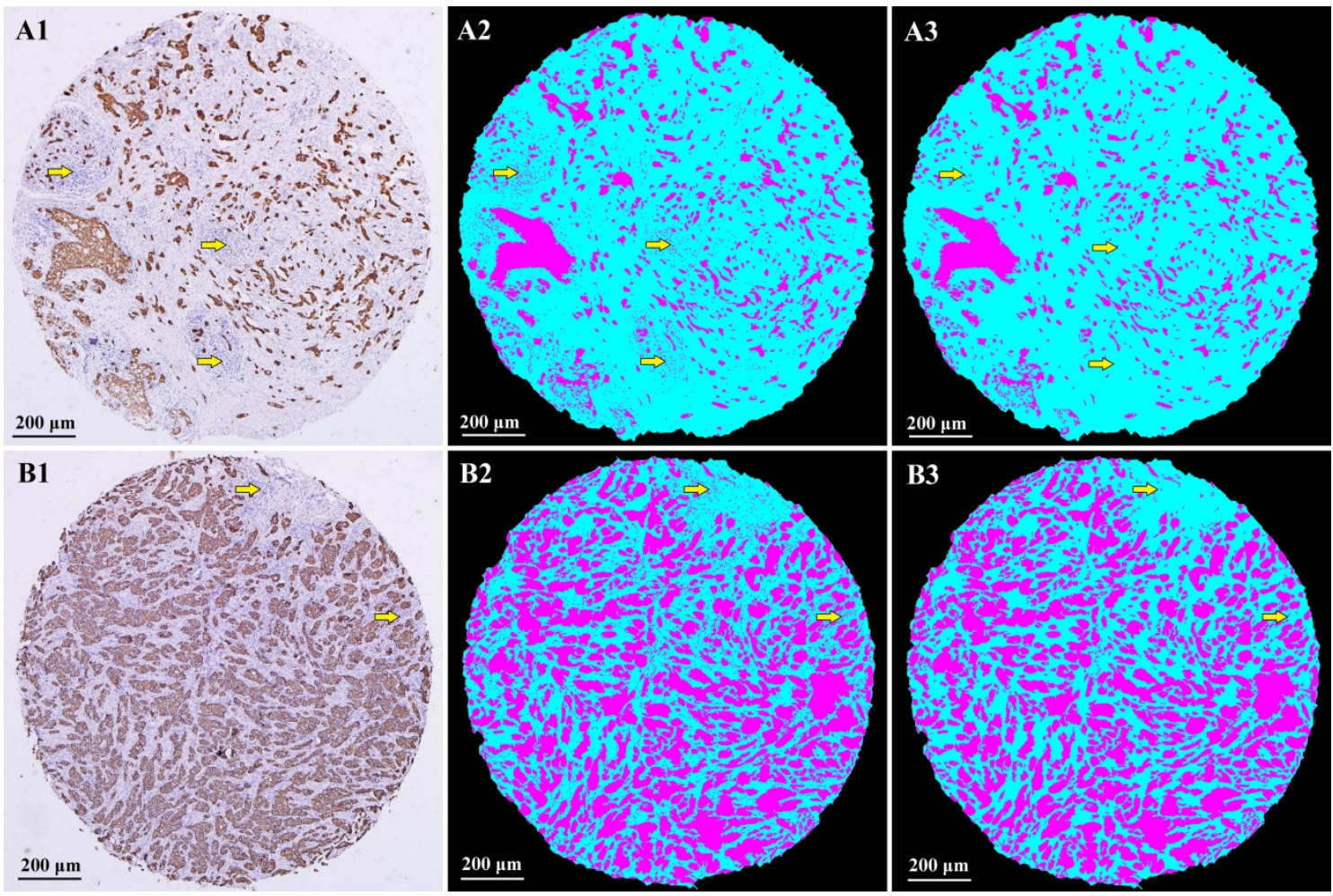

Figure 8. Comparison of recognition results by different thresholds. Panels A2, B2, a lower threshold was set, part of the cell nuclei of stroma cells stained in blue by hematoxylin were mistakenly identified as tumor objects. Panels A3, B3, a suitable threshold was set to eliminate small objects like the cell nuclei.

\section{Supplementary Material}

Supplementary figures and tables.

http://www.jcancer.org/v12p3427s1.pdf

\section{Acknowledgements}

This work was supported by the Young Scientists Fund of National Natural Science Foundation (Grant Numbers 81701768 and 81702901).

\section{Ethical statement}

Approval of the study protocol was obtained from the Institutional Ethics Committee of Zhongnan Hospital of Wuhan University (Scientific Ethical Approval No. [2017057]). The study was undertaken according to the ethical standards of the World Medical Association Declaration of Helsinki.

\section{Competing Interests}

The authors have declared that no competing interest exists.

\section{References}

1. Bray F, Ferlay J, Soerjomataram I, et al. Global cancer statistics 2018: GLOBOCAN estimates of incidence and mortality worldwide for 36 cancers in 185 countries. CA Cancer J Clin. 2018; 68(6):394-424.

2. Rui-Mei Feng, Yi-Nan Zong, Su-Mei Cao, et al. Current cancer situation in China: good or bad news from the 2018 Global Cancer Statistics. Cancer Commun (Lond). 2019; 39(1):22.

3. Li N, Deng Y, Zhou L, et al. Global burden of breast cancer and attributable risk factors in 195 countries and territories, from 1990 to 2017: results from the Global Burden of Disease Study 2017. J Hematol Oncol. 2019; 12(1):140.
4. Ellington TD, Henley SJ, Wilson RJ, et al. Breast Cancer Survival Among Males by Race, Ethnicity, Age, Geographic Region, and Stage - United States, 2007-2016. MMWR Morb Mortal Wkly Rep. 2020; 69(41):1481-1484.

5. Ting $\mathrm{Wu}$, Yun Dai. Tumor microenvironment and therapeutic response. Cancer Lett. 2017: 387:61-68.

6. Denton AE, Roberts EW, Fearon DT. Stromal Cells in the Tumor Microenvironment. Adv Exp Med Biol. 2018; 1060:99-114.

7. Ngambenjawong C, Gustafson HH, Pun SH. Progress in tumor-associated macrophage (TAM)-targeted therapeutics. Adv Drug Deliv Rev. 2017; 114:206-221.

8. Zielińska KA, Katanaev VL. The Signaling Duo CXCL12 and CXCR4: Chemokine Fuel for Breast Cancer Tumorigenesis. Cancers (Basel). 2020; 12(10):E3071.

9. C J H Kramer, K M H Vangangelt, G W van Pelt, et al. The prognostic value of tumour-stroma ratio in primary breast cancer with special attention to triple-negative tumours: a review. Breast Cancer Res Treat. 2019; 173(1):55-64.

10. Vangangelt KMH, Green AR, Heemskerk IMF, et al. The prognostic value of the tumor-stroma ratio is most discriminative in patients with grade III or triple-negative breast cancer. 2020; 146(8):2296-2304.

11. Qian Xu, Jing-Ping Yuan, Yuan-Yuan Chen, et al. Prognostic Significance of the Tumor-Stromal Ratio in Invasive Breast Cancer and a Proposal of a New Ts-TNM Staging System. J Oncol. 2020; 2020:9050631.

12. Vinod P Balachandran, Mithat Gonen, J Joshua Smith, et al. Nomograms in oncology: more than meets the eye. Lancet Oncol. 2015;16(4):e173-80.

13. Sun Young Kim, Min Joo Yoon, Young Iee Park, et al. Nomograms predicting survival of patients with unresectable or metastatic gastric cancer who receive combination cytotoxic chemotherapy as first-line treatment. Gastric Cancer. 2018; 21(3):453-463.

14. Chen C, Sun SR, Gong YP, et al. Quantum dots-based molecular classification of breast cancer by quantitative spectroanalysis of hormone receptors and HER2. Biomaterials. 2011; 32(30):7592-9.

15. Jing Ping Yuan, Lin Wei Wang, Ai Ping Qu, et al. Quantum dots-based quantitative and in situ multiple imaging on ki67 and cytokeratin to improve ki67 assessment in breast cancer. PLoS One. 2015; 10(4):e0122734.

16. Mahul BA, Stephen E, Frederick G, David RB, Robert K. AJCC Cancer Staging Manual (ed 8), 8th ed. 2017. Springer, New York

17. Lakhani SR, Ellis IO, Schnitt SJ, Tan PH, van de Vijver MJ. WHO classification of tumours of the breast. World Health Organization classification of tumours, 4th ed. 2012. IARC, Lyon

18. Chen $\mathrm{C}$, Xia HS, Gong YP et al. The quantitative detection of total HER2 load by quantum dots and the identification of a new subtype of breast cancer with different 5-year prognosis. Biomaterial. 2010;31(33):8818-25.

19. Hammond ME, Hayes DF, Wolff $\mathrm{AC}$, et al. American society of clinical oncology/college of american pathologists guideline recommendations for 
immunohistochemical testing of estrogen and progesterone receptors in breast cancer. J Clin Oncol. 2010; 28(16):2784-95.

20. Wolff AC, Hammond ME, Hicks DG, et al. Recommendations for human epidermal growth factor receptor 2 testing in breast cancer: American Society of Clinical Oncology/College of American Pathologists clinical practice guideline update. J Clin Oncol. 2013; 31(31):3997-4013.

21. Mesker WE, Junggeburt JM, Szuhai $K$, et al. The carcinoma-stromal ratio of colon carcinoma is an independent factor for survival compared to lymph node status and tumor stage. Cell Oncol. 2007; 29(5):387-98.

22. Vangangelt $\mathrm{KMH}$, van Pelt $\mathrm{GW}$, Engels $\mathrm{CC}$, et al. Prognostic value of tumor-stroma ratio combined with the immune status of tumors in invasive breast carcinoma. Breast Cancer Res Treat. 2018; 168(3):601-612.

23. West NP, Dattani M, McShane P, et al. The proportion of tumour cells is an independent predictor for survival in colorectal cancer patients. Br J Cancer. 2010; 102(10):1519-23.

24. Downey CL, Simpkins SA, White J, et al. The prognostic signifcance of tumour-stroma ratio in oestrogen receptor-positive breast cancer. Br J Cancer. 2014; 110(7):1744-1747.

25. Roeke T, Sobral-Leite M, Dekker TJA, et al. The prognostic value of the tumour-stroma ratio in primary operable invasive cancer of the breast: a validation study. Breast Cancer Res Treat. 2017; 166(2):435-445.

26. Downey CL, Thygesen HH, Sharma N, et al. Prognostic signifcance of tumour stroma ratio in inflammatory breast cancer. Springerplus. 2015; 4:68

27. Dundar MM, Badve S, Bilgin G, et al. Computerized classification of intraductal breast lesions using histopathological images. IEEE Trans Biomed Eng. 2011; 58(7):1977-84.

28. Bo Li, Yang Wang, Hui Jiang, et al. Pros and Cons: High Proportion of Stromal Component Indicates Better Prognosis in Patients With Pancreatic Ductal Adenocarcinoma-A Research Based on the Evaluation of Whole-Mount Histological Slides. Front Oncol. 2020; 10:1472.

29. Jun $\mathrm{Xu}$, Xiaofei Luo, Guanhao Wang, et al. A Deep Convolutional Neural Network for segmenting and classifying epithelial and stromal regions in histopathological images. Neurocomputing. 2016;191:214-223.

30. de Kruijf EM, van Nes JG, van de Velde CJ, et al. Tumor-stroma ratio in the primary tumor is a prognostic factor in early breast cancer patients, especially in triple-negative carcinoma patients. Breast Cancer Res Treat. 2011; 125(3):687-96.

31. Moorman AM, Vink $\mathrm{R}$, Heijmans $\mathrm{HJ}$, et al. The prognostic value of tumour-stroma ratio in triple-negative breast cancer. Eur J Surg Oncol. 2012; 38(4):307-13.

32. Dekker TJ, van de Velde CJ, van Pelt GW, et al. Prognostic significance of the tumor-stroma ratio: validation study in node-negative premenopausal breast cancer patients from the EORTC perioperative chemotherapy (POP) trial (10854). Breast Cancer Res Treat. 2013; 139(2):371-9.

33. Gujam FJ, Edwards J, Mohammed ZM, et al. The relationship between the tumour stroma percentage, clinicopathological characteristics and outcome in patients with operable ductal breast cancer. Br J Cancer. 2014; 111(1):157-65

34. Chen C, Sun S, Yuan JP, et al. Characteristics of breast cancer in Central China, literature review and comparison with USA. Breast. 2016; 30:208-213.

35. Li T, Mello-Thoms C, Brennan PC. Descriptive epidemiology of breast cancer in China: incidence, mortality, survival and prevalence. Breast Cancer Res Treat. 2016; 159(3):395-406.

36. Gupta R, Sinha S, Paul RN. The impact of microsatellite stability status in colorectal cancer. Curr Probl Cancer. 2018; 42(6):548-559.

37. Kawakami H, Zaanan A, Sinicrope FA. Microsatellite instability testing and its role in the management of colorectal cancer. Curr Treat Options Oncol. 2015; 16(7):30.

38. He C, Sun S, Zhang Y, et al. A Novel Nomogram to Predict Survival in Patients With Recurrence of Pancreatic Ductal Adenocarcinoma After Radical Resection. Front Oncol. 2020;10:1564.

39. Li B, Jiang C, Wang R, et al. Prognostic Value of a Nomogram Based on the Dynamic Albumin-to-Alkaline Phosphatase Ratio for Patients with Extensive-Stage Small-Cell Lung Cancer. Onco Targets Ther. 2020; 13:9043-9057. 